\title{
Oui ou non pour le dépistage de la violence familiale dans les salles d'urgence?
}

\author{
Kathleen Mackay, M.S.S., t.s.a.
}

Voir article connexe à la PAge 325

ENGLISH VERSION ON PAGE 329

$I_{1}^{1}$ 1 est grand temps d'aborder la grave question de la violence conjugale, un problème persistant de santé publique, et l'étude de McClennan et de ses collègues figurant dans ce numéro du JCMU, «Caring for Victims of Intimate Partner Violence: A Survey of Canadian Emergency Departments "1, attire l'attention sur ce sujet. La violence conjugale est une sous-catégorie de la violence familiale, qui englobe notamment la violence envers les personnes âgées et la violence envers les enfants. Chaque année, la violence conjugale affecte la santé de milliers de personnes en Amérique du Nord, tout particulièrement celle des femmes. Malgré l'ampleur du problème, les auteurs ont constaté que le nombre de salles d'urgence s'étant dotées de politiques concernant la violence conjugale n'avait pas augmenté de façon significative sur une période de 10 ans et depuis la publication en 2003 des lignes directrices du Groupe d'étude canadien sur les soins de santé préventifs. Les auteurs ont signalé qu'en raison du manque de preuves concernant l'efficacité des interventions, les groupes de travail aux États-Unis et au Canada n'ont pas pris position pour ou contre le dépistage de la violence conjugale. Ils avancent néanmoins qu'il serait probablement bénéfique que les fournisseurs de soins de santé repèrent les cas de violence entre conjoints et qu'ils réagissent de façon à maximiser les avantages et à éviter que d'autres actes violents ne soient commis contre la victime.

En dépit de notre ambivalence générale quant au dépistage systématique de la violence conjugale, il importe de signaler qu'une telle action pourrait comporter d'importants avantages. Mon expérience des 15 dernières années en tant que coordonnatrice des programmes de violence familiale dans deux grands hôpitaux universitaires et une clinique externe de violence familiale m'a permis de constater que les consultations à l'urgence sont des occasions de sensibiliser la population à l'impact de la violence familiale sur la santé, de poser des questions sur la sécurité des relations pour les patients et de fournir des renseignements sur les ressources offertes dans les hôpitaux et la collectivité pour aider les victimes et les agresseurs.

Lancé en 1992 grâce à une subvention de Santé Canada, le programme de violence familiale à l'Hôpital général de Vancouver était parmi les premiers programmes de ce genre au Canada. Il visait à élaborer une stratégie de soins pour repérer les victimes de violence conjugale chez les femmes se présentant à l'urgence, les évaluer, les traiter, les référer et exercer un suivi à leur égard. Nous avons mis en application le dépistage systématique, par les infirmières, de toutes les patientes se présentant à l'urgence et, lorsqu'un cas de violence conjugale était divulgué, des protocoles précis de prise en charge et de documentation par une équipe interdisciplinaire devaient être observés. Auparavant, nos données indiquaient que très peu de cas de violence conjugale étaient détectés à l'urgence. Or, depuis le lancement du programme, nous nous sommes entretenus avec des milliers de patientes victimes de violence conjugale, les avons écoutées, avons validé leurs situations, les avons encouragées à utiliser les ressources disponibles et leur avons fourni de la documentation. Nous procédons désormais au dépistage de tous les adultes, hommes ou femmes, dans la salle d'urgence et à la clinique de préadmission de l'Hôpital général de Vancouver ainsi que par l'intermédiaire des services de soins de santé

Coordonnatrice clinique, Programme de violence familiale et clinique externe, Hôpital général de Vancouver et Providence Health Care, Vancouver, C.-B.

Les opinions exprimées dans les éditoriaux sont celles des auteurs; elles ne reflètent pas nécessairement celles du JCMU ou de l'Association canadienne des médecins d'urgence.

JCMU 2008;10(4):331-2 
Providence Health Care, qui comprennent l'hôpital St. Paul's et l'hôpital Mount Saint Joseph.

Le programme de violence familiale de l'Hôpital général de Vancouver et d'autres programmes semblables font la promotion de la santé, d'une part, en accroissant la sensibilisation à l'impact de la violence conjugale sur la santé et, d'autre part, en offrant une intervention précoce aux personnes touchées. Lorsque l'on pose des questions sur les mauvais traitements, on sème des graines d'espoir chez les personnes qui nient le mauvais traitement, en sachant que certaines reviendront plus tard pour obtenir de l'aide. On apprend à d'autres, qui ont peut-être des amis ou des membres de leur famille vivant des situations de violence, où les victimes de violence familiale peuvent obtenir de l'aide. À cet égard, le simple fait de poser des questions constitue en soi une intervention potentiellement bénéfique. Le dépistage de cas de violence conjugale continue néanmoins de soulever la controverse. Ses causes et les interventions possibles sont complexes. Il n'est donc pas surprenant que les groupes de travail aux États-Unis et au Canada n'aient pas défini jusqu'à maintenant d'interventions précises dont l'efficacité a été prouvée. Ils ne sont donc pas en mesure de recommander ou de déconseiller le dépistage de la violence conjugale.

En outre, la violence conjugale constitue un problème social et de santé complexe qui met en cause une autre personne, nommément l'agresseur, dont on ne contrôle pas les actions. Bien que le principal objet des interventions en cas de violence conjugale soit d'assurer la sécurité de la victime, bon nombre de reportages médiatiques soulignent le fait que l'agresseur qui cherche à faire du tort à son ex-conjoint ou aux membres de sa famille ou même à les tuer arrive souvent à ses fins.

Pendant des décennies, les agences communautaires ont aidé les victimes de violence familiale et géré des programmes pour les agresseurs. En revanche, les agences de santé ont pris du temps à réagir à cette question. McClennan et ses collègues ${ }^{1}$ estiment que la mise en place de politiques et de procédures qui répondent aux besoins des patients exposés à la violence conjugale devrait être une priorité. Je suis tout à fait d'accord. Mais n'attendons toutefois pas l'impossible, c'est-à-dire une intervention coulée dans «un moule unique » dont l'efficacité a été prouvée et qui par conséquent peut justifier le dépistage de la violence conjugale. Il n'existe tout simplement pas de remède universel contre ce mal social et communautaire qui représente un risque pour la santé.

À bien des égards, la violence conjugale s'apparente à la conduite en état d'ivresse où la perception publique et les normes communautaires peuvent influencer les comportements. L'attention médiatique accordée à l'impact de l'alcool au volant sur ses victimes a mené à l'élaboration de bon nombre de stratégies, dont la consommation responsable d'alcool, le recours à un conducteur désigné et l'utilisation de taxis. La violence familiale n'a pas bénéficié de campagnes de sensibilisation soutenues visant à modifier le comportement des agresseurs. Qui plus est, la majeure partie des agresseurs n'ont pas véritablement conscience de l'impact profond que leurs actions ont sur les autres. Or, nous ne pouvons songer à résoudre le problème de la violence conjugale sans nous attaquer avant tout au comportement de l'agresseur. La sensibilisation du public à la violence en milieu familial et à son impact sur la famille est un élément essentiel de cette solution. J'estime que nos salles d'urgence peuvent jouer un rôle de premier plan dans cette démarche. Elles peuvent à tout le moins apposer des affiches et renseigner les patients sur les mauvais traitements et les ressources communautaires auxquelles peuvent avoir recours les victimes et les agresseurs. Par surcroît, même si ses avantages n'ont pas été prouvés, je suis d'avis que le dépistage systématique a sa place dans nos salles d'urgence. Pourquoi? Parce que la victime y est déjà et qu'il est fort probable qu'elle revienne à l'urgence si elle continue de subir de mauvais traitements. Pendant la brève période où l'on voit les patients à l'urgence, les actions suivantes sont envisageables : cerner la nature du problème, réitérer à la victime qu'elle n'est pas responsable des actes qu'elle subit, l'encourager à adopter des comportements sécuritaires et documenter les situations. Ces mesures pourraient être extrêmement efficientes, car on est déjà en présence du patient et l'intervention est principalement de nature empathique et informative. Poser des questions sur la violence conjugale ouvre la porte à une guérison bienfaisante. Cela peut également réduire la morbidité et la mortalité liées à ce grave problème de santé publique.

\section{Conflits d'intérêts : aucun déclaré.}

Mots clés : violence familiale, violence conjugale, salle d'urgence, dépistage, sensibilisation du public

\section{Références}

1. McClennan S, Worster A, MacMillan H. Caring for victims of intimate partner violence: a survey of Canadian emergency departments. JCMU 2008;10:325-8.

Correspondance : Kathleen Mackay, Programme de violence familiale, Hôpital général de Vancouver, 855 12e Avenue Ouest, Vancouver BC V5Z1M9; kathleen.mackay@vch.ca 